\title{
An Empirical Approach - Distributed Mobility Management for Target Tracking in MANETs
}

\author{
Mr. N. Kumaresh \\ Lecturer \\ Department of MCA \\ Sri Ramakrishna Institute of Technology \\ Coimbatore-10 \\ Tamilnadu (India)
}

\begin{abstract}
Mobility management is a major challenge in mobile ad hoc networks (MANETs) due in part to the dynamically changing network topologies. For mobile sensor networks that are deployed for surveillance applications, it is important to use a mobility management scheme that can empower nodes to make better decisions regarding their positions such that strategic tasks such as target tracking can benefit from node movement. In this paper, we describe a distributed mobility management scheme for mobile sensor networks. The proposed scheme considers node movement decisions as part of a distributed optimization problem which integrates mobility-enhanced improvement in the quality of target tracking data with the associated negative consequences of increased energy consumption due to locomotion, potential loss of network connectivity, and loss of sensing coverage.
\end{abstract}

Key Words- Mobile Adhoc Networks, Target Tracking, Mobility Management

\section{INTRODUCTION}

Mobility management has long been recognized major challenge in mobile ad hoc networks. Mobility management is a major challenge in mobile ad hoc networks (MANETs) due in part to the dynamically changing network topologies.

Mobility management in sensor networks is different from that in mobile ad hoc networks because the movement of sensor nodes here is not random; rather, the movement of sensor nodes is purposeful, e.g., to actively and better track an intruder. The MANET has the following characteristics:

1. New member can join and leave the network any time.

2. No base station is available to provide connectivity

3. It is difficult to implement sophisticated scheme for handover and location management

4. Each node act as a router, forwarding packets from other nodes

In such scenarios, it is important to have an efficient mobility management scheme to ensure that sensor node mobility is exploited in the best possible way, e.g., to improve the quality of target tracking. At the same time, the mobility management strategy should avoid inefficient usage of scarce resources, such as energy and network bandwidth.

Furthermore, the mobility management scheme should also take into account the potential negative consequences of node movement, e.g., loss of area coverage, loss of connectivity, and degradation of network performance. In addition, node movement also involves locomotion energy and routing overhead, especially the need to reestablish routes.

\section{Related Works}

Recent research efforts on target tracking in wireless sensor networks have focused on collaborative sensing energy-efficient routing and management and sensor node deployment. Collaborative sensing and signal processing provide raw sensory data from the low-level sensing units on sensor nodes. In many cases, cheap sensors such as omni directional acoustic sensors are used since alternatives such as CCD cameras generally require more resources for power, memory, bandwidth, and computation.

We focus on the mobility management problem for mobile sensor networks in this project. Mobility management in sensor networks is different from that in mobile ad hoc networks because the movement of sensor nodes here is not random; rather, the movement of sensor nodes is purposeful, e.g., to actively and better track an intruder. In such scenarios, it is important to have an efficient mobility management scheme to ensure that sensor node mobility is exploited in the best possible way, e.g., to improve the quality of target tracking. At the same time, the mobility management strategy should avoid inefficient usage of scarce resources, such as energy and network bandwidth.

Furthermore, the mobility management scheme should also take into account the potential negative consequences of node movement, e.g., loss of area coverage, loss of connectivity, and degradation of network performance. In addition, node movement also involves locomotion energy and routing overhead, especially the need to reestablish routes. Although the target information from a single node is generally limited, more useful information can be obtained through data exchange and aggregation between multiple nodes, based upon which higherlevel strategic decisions can be made. 
Routing in ad hoc sensor networks has received a lot attention and is considered a great challenge for ad hoc sensor networks. Many efforts have been made to achieve energy-efficient routing in data aggregation, especially for target tracking applications. The LEACH protocol forms a clustered hierarchy in sensor networks, where the cluster head will be responsible for transmitting sensor data for its cluster members.

The energy savings is achieved because the data is consolidated through such clusterization. SPAN is another energy efficient routing protocol where sensor nodes are selected to operate on off-duty and on-duty cycles for sensor nodes. We present an analysis that evaluates the risks of losing connectivity and sensing coverage from the perspective of a mobile sensor network with an inherently dynamic topology.

A mobility management framework that unifies tracking quality, sensing coverage, network connectivity, and energy consumption is introduced. Finally, we present a distributed algorithm for implementing the proposed mobility management scheme.

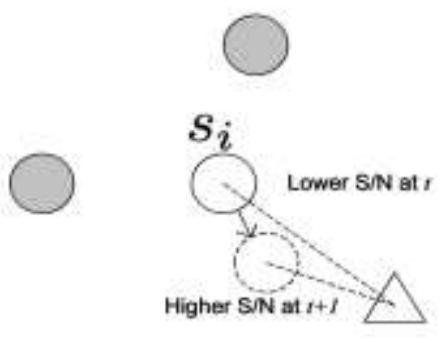

(a)

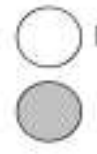

Mobile sensor node $s i$ at time

One-hop neighbors of mobile sensor node si at time $t$

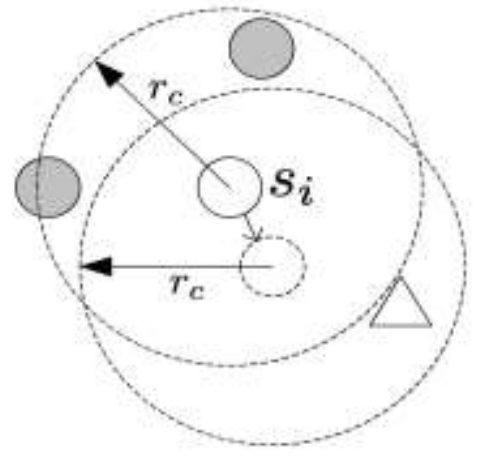

(b)

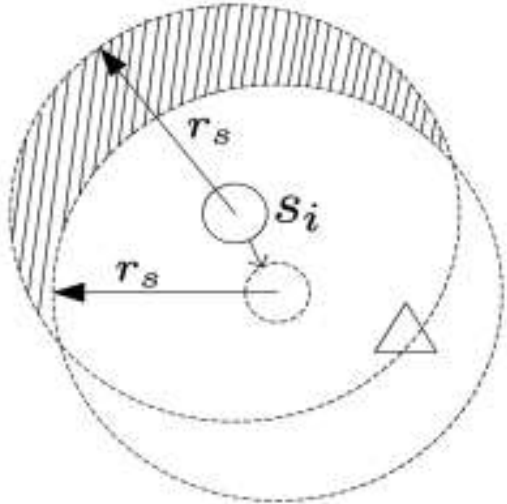

(c)

Fig: (a) To improve tracking quality, mobile node Si chooses to move to a location that is expected to have a higher signal-to-noise ratio. Fig (b) Movement of Si may break the communication connectivity with its neighbor nodes. Fig (c) Movement of Si may cause some area to under coverage at $\mathrm{t}$ time to become uncovered at $\mathrm{t}+1$.

Computation capabilities for centralized processing; all other nodes forward their collected sensor data. The second approach depends on a dynamic clustering algorithm to select one of the nodes as the cluster head, i.e., the node that performs sensor fusion. When the cluster head is changed, usually in accordance with the estimated target track, it needs to pass the prior information to the next cluster head for continuous tracking.

Mobile ad hoc networks have received considerable attention in the literature. Most existing methods for mobility management focus on communication issues arising from dynamically changing topologies due to node mobility.

\section{Assumptions}

To simplify the discussions, we make the following assumptions for the sensor network:

1. In this paper, we assume that both sensor nodes and the target are moving at constant speeds.

2. We assume that the sampling interval of all sensor nodes is small enough such that there is no drastic change in sensor measurements of the target state.
3. All nodes have the same number of candidate locations where thay can move.

4. A node uses the prior of its current location to predict the sensor measurements at its candidate locations.

5. A node uses the current sensor measurements from its current one-hop neighbor nodes.

\section{Finding Best Move for a Node to Improve Quality of Target Tracking}

To improve the quality of target tracking, a node can decide to move to another location at the next time instant. These locations are referred to as candidate locations. Then formulate the problem of selecting the best candidate location for a node in a fully distributed manner.

\section{Estimates of Negative Consequences}

Estimates of negative consequences focuses on the energy, connectivity and coverage issues. Nodes have to spend additional energy for movement. Even though sensor nodes on mobile platforms can carry more battery supplies it is important to 
ensure that the available energy is properly used to best serve the purpose of surveillance tasks.

\section{Decisions on Node Movement}

Decisions on node movement involve the decision on node movement using Cost evaluation, decision on movement and analysis of time complexity.

The selection rule based on the cost evaluation that takes into account all negative consequences due to move movement.
Nodes can exchange their expected total cost and decide who should move. When the total cost is obtained for all candidate locations, the optimal selection of the candidate location for node can be obtained by considering both positive and negative consequences.

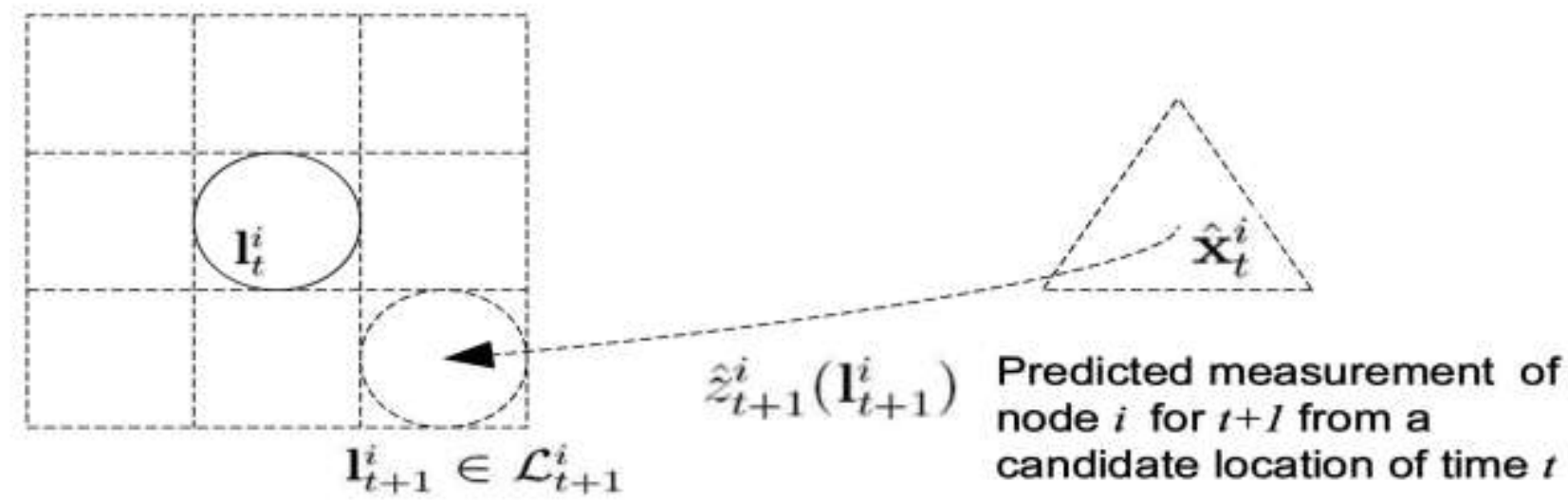

(

Mobile sensor node $i$ at time $t$

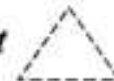

Target from local estimate of node $i$ Mobile sensor node $i$ at candidate location of time $t+1$

Fig: Node $S_{i}$ predicts its measurement at a candidate location based on its current target estimate

\section{Simulated Studies}

\subsection{Static Sensor Network versus Mobile Network}

\section{with Mobility Management}

The selection of the candidate locations for target tracking data improvement is based on the trace of the error covariance matrix. The error for a mobile sensor network is less than that for the static network. Another well-accepted metric for evaluating the tracking quality is the norm of the position error. The norm of the position error for the mobile network is roughly 72.5 percent less during the time that target is moving through the sensor field.

The average global coverage is defined as the sum of individual grid points over the total number of grid points. The mobility management scheme improves the global coverage compared to the static network. Every node can make its movement decision in a timely manner for dynamic target tracking without lengthy negotiation with neighbors for maintaining connectivity and sensing coverage. This ensures a flexible distributed implementation for mobility management in mobile sensor networks fro target tracking.
The performance of the proposed scheme is related to factors such as the speed of the nodes, the target speed and the number of nodes deployed in the sensing region. When the number of nodes deployed increases, the target tracking quality is improved because more sensor data are available within the one-hop neighborhood for the node to make movement decisions based on local knowledge.

\section{Results}

Each node decides to move to reach their target by estimating the consequences such as update count, clustering speed, interclustering distance, and communication cost.. Based upon the clustering speed, inter-clustering distance, update count and communication cost nodes decide where to move.

The result shows the performance of the proposed approach distributed mobility approach. Method 1 in the figure result of brute force mobility shows the result after applying the brute force mobility in the network. 


Result
Result
Result [60 Objects, Method: 1]:
UpdateCount: 1200.0
Communication Cost: $2040.0 \mathrm{~kb}$
Awg. InterClusterDistance: 192.16092386914414
ClusteringSpeed: $480.0 \mathrm{~s}$

Fig: Result of Brute Force Mobility

Method 2 in the figure result of existing distributed mobility approach shows the result of existing distributed mobility approach. The result shows the difference in the communication cost, update count. Average inter-clustering distance and clustering speed.

\begin{tabular}{l}
\hline Result \\
\hline Result \\
Result [60 Objects, Method: 1]: \\
UpdateCount: 1200.0 \\
Communication Cost: $2040.0 \mathrm{~kb}$ \\
Avg.InterClusterDistance: 193.9453720527236 \\
ClusteringSpeed: $480.0 \mathrm{~s}$ \\
Result [60 Objects, Method: 2 ]: \\
UpdateCount: 199.0 \\
Communication Cost: $338.3 \mathrm{~kb}$ \\
Avg.InterClusterDistance: 230.56257686778358 \\
ClusteringSpeed: $79.6 \mathrm{~s}$
\end{tabular}

Fig: Result of Existing Distributed Mobility Approach

Method 3 in the figure result of proposed distributed mobility approach shows the result of existing distributed mobility approach. The result shows the difference in the communication cost, update count. Average inter-clustering distance and clustering speed. All the three results show that the proposed distributed mobility approach has less negative consequences.

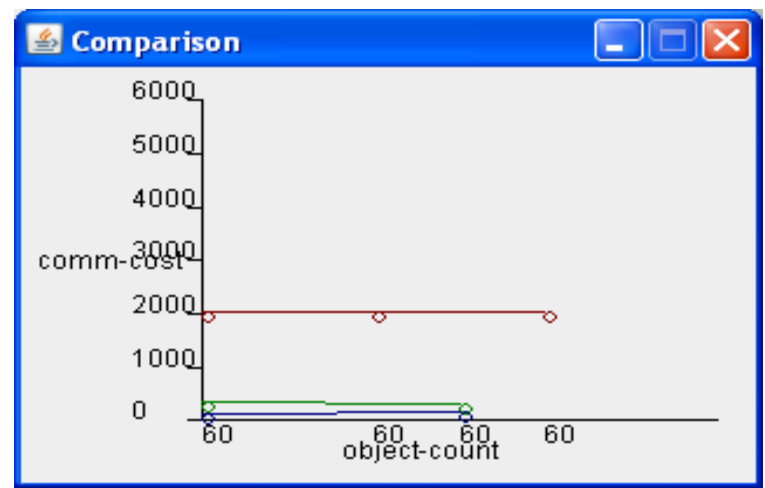

Fig: Comparison on Communication Cost

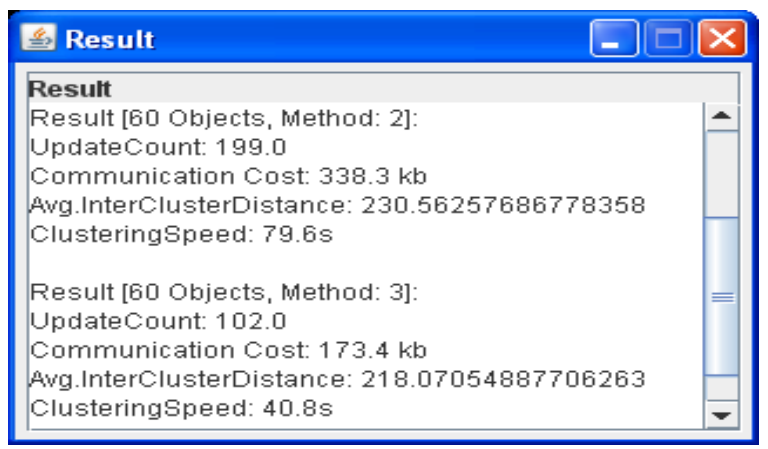

Fig: Result of Proposed Distributed Mobility Approach

\section{Comparisons}

Comparisons are done on the clustering speed, inter-clustering distance, update-count and communication cost. The comparison on the consequences such as update count, clustering speed, inter-clustering distance and communication cost are shown as a chart. The comparison helps to make an evaluation of the performance of the node movement in existing system and proposed system as well as it shows how the static sensor network differs from mobile sensor network. The charts shown below describe the negative consequences between the existing approach and proposed approach. It also describes the enhancement of the proposed approach.

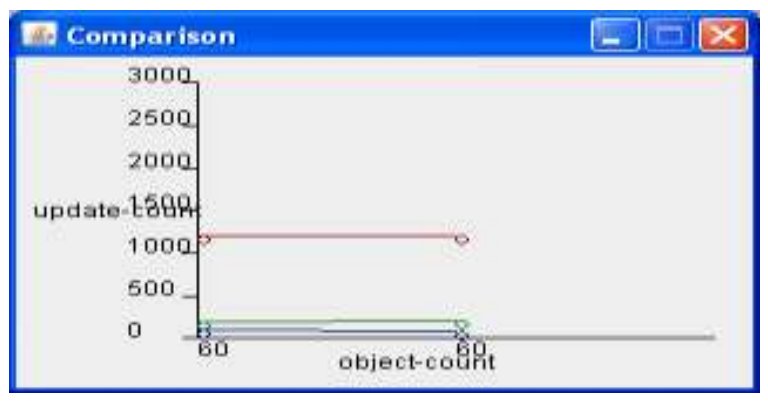

Fig: Comparison on Update-Count

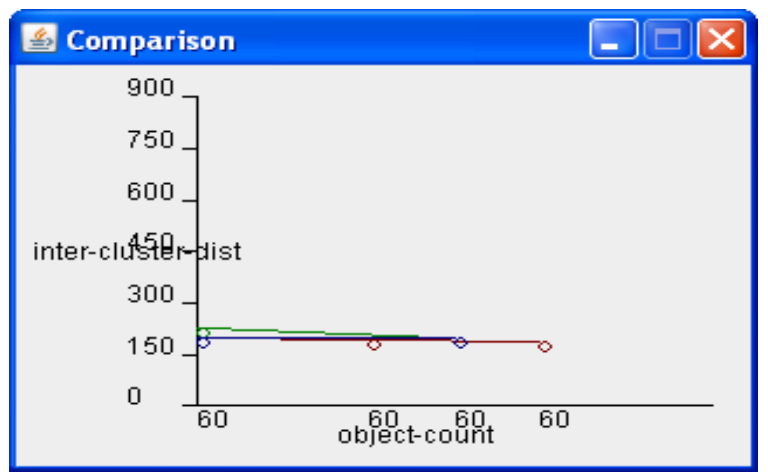

Fig: Comparison on Inter-Clustering Distance 


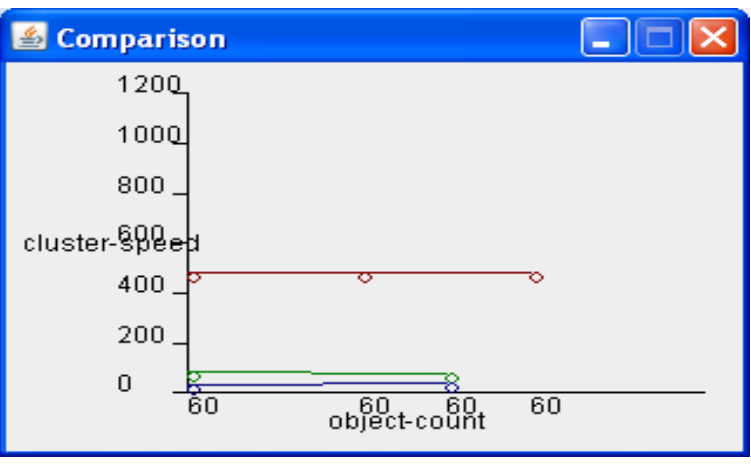

Fig: Comparison on Cluster Speed

\section{Conclusions}

Mobility management scheme for mobile sensor networks considers target tracking quality, connectivity breakage, and loss of sensing coverage and energy consumption due to node movement. The constantly changing topology due to node movement makes mobility management difficult for mobile sensor networks. The cost evaluation technique allows us to trade off target tracking quality improvement with the negative consequences of energy consumption, loss of connectivity and coverage.

\section{References}

[1] Zou and Chakrabary, "Distributed Mobility Management for Target Tracking in Mobile sensor Networks," IEEE Mobile Computing, pp 872-887, 2007.

[2] I.F. Akyildiz, W. Su, y. Sankarasubramaniam, and E. CAyirci, "A Survey on Sensor Networks," IEEE Comm. Magazine, pp. 102-114, Aug. 2002.

[3] F.Baker, “An Outsider's view of MANET,'IETF Internet draft, work in progress, June 2006.

[4] R.R.Brooks, C.Griffin, and D.Friedlander, "Distributed Target Classsification and tracking in sensor networks," proc. IEEE, pp. 1163 -1171, 2003.

[5] T.Camp, J.Boleng, and V.Davies, "A Survey of Mobility Models for Ad Hoc Network Research," J.Wireless Comm. Mobile Computing, vol.2, pp.483-502, 2002.

[6] S.S. Iyengar and R.R. Brooks, Handbook on Distributed Sensor Networks. Chapman and Hall/CRC Press, July 2003. 